\title{
Highwire Regulates Guidance of Sister Axons in the Drosophila Mushroom Body
}

\author{
Jung Eun Shin and Aaron DiAntonio \\ Department of Developmental Biology, Hope Center for Neurological Disorders, Washington University School of Medicine, St. Louis, Missouri 63110
}

Axons often form synaptic contacts with multiple targets by extending branches along different paths. PHR (Pam/Highwire/RPM-1) family ubiquitin ligases are important regulators of axon development, with roles in axon outgrowth, target selection, and synapse formation. Here we report the function of Highwire, the Drosophila member of the PHR family, in promoting the segregation of sister axons during mushroom body (MB) formation. Loss of highwire results in abnormal development of the axonal lobes in the MB, leading to thinned and shortened lobes. The highwire defect is attributable to guidance errors after axon branching, in which sister axons that should target different lobes instead extend together into the same lobe. The highwire mutant MB displays elevation in the level of the MAPKKK Wallenda/DLK (dual leucine zipper kinase), a previously identified substrate of Highwire, and genetic suppression studies show that Wallenda/DLK is required for the highwire MB phenotype. The highwire lobe defect is limited to $\alpha / \beta$ lobe axons, but transgenic expression of highwire in the pioneering $\alpha^{\prime} / \beta^{\prime}$ neurons rescues the phenotype. Mosaic analysis further shows that $\alpha / \beta$ axons of highwire mutant clones develop normally, demonstrating a non-cell-autonomous role of Highwire for axon guidance. Genetic interaction studies suggest that Highwire and Plexin A signals may interact to regulate normal morphogenesis of $\alpha / \beta$ axons.

\section{Introduction}

Axonal navigation to distant targets is finely controlled by factors regulating the motility of the axon tip, the direction of axon extension, and branching of axons. The Drosophila mushroom body (MB) participates in olfactory-associative learning and is a powerful model system to investigate such axonal behaviors (Heisenberg, 1998; Davis, 2005; Fahrbach, 2006). The MB is composed of three neuronal subtypes $-\gamma, \alpha^{\prime} / \beta^{\prime}$, and $\alpha / \beta$ neurons- that can be distinguished by their birth order and the location of their axonal projections (Lee et al., 1999). Cell bodies of these neurons are located in the posterior cortex, and they extend axons through a tract called the peduncle. The axons then bifurcate and send sister branches into a dorsal and a medial lobe in which the axons synapse onto their targets. Because laterdeveloping axons grow along the earlier-developing axons within the pathway, non-cell-autonomous regulation is likely to be important for the guidance of these follower axons (Araújo and Tear, 2003; Fushima and Tsujimura, 2007).

PHR (Pam/Highwire/RPM-1) proteins are a conserved family of E3 ubiquitin ligases with essential roles in axon development (Schaefer et al., 2000; Wan et al., 2000; Zhen et al., 2000; D’Souza et al., 2005; Bloom et al., 2007; Lewcock et al., 2007). The PHR

\footnotetext{
Received July 29, 2011; revised Sept. 30, 2011; accepted 0ct. 11, 2011.

Author contributions: J.E.S. and A.D. designed research; J.E.S. performed research; J.E.S. and A.D. wrote the paper.

This work was supported by National Institutes of Health Grant DA020812 (A.D.). We are grateful to members of the DiAntonio laboratory for helpful discussions and Xiaolu Sun for technical support. We also thank the Bloomington Stock Center, the Drosophila Genetic Resource Center, and the Vienna Drosophila RNAi Center for fly lines.

The authors declare no competing financial interests.

Correspondence should be addressed to Aaron DiAntonio, Department of Developmental Biology, Campus Box 8103, Washington University School of Medicine, St. Louis, M0 63110. E-mail: diantonio@wustl.edu.

DOI:10.1523/JNEUROSCI.3902-11.2011

Copyright $\odot 2011$ the authors $\quad 0270-6474 / 11 / 3117689-12 \$ 15.00 / 0$
}

family comprises mouse Phr1, human Pam, zebrafish Esrom, Drosophila Highwire (Hiw), and Caenorhabditis elegans RPM-1, which share a RING finger domain with ligase activity (Po et al., 2010). In Drosophila and C. elegans, PHR regulates synapse development at the axon terminal by downregulating the MAP kinase kinase kinase (MAPKKK) Wallenda (Wnd)/DLK (dual leucine zipper kinase) to restrict MAPK signaling (Nakata et al., 2005; Collins et al., 2006). Mutants of vertebrate PHR exhibit dramatic axonal defects, including loss of axon tracts, mistargeting of axons, and abnormal growth cone morphology (Bloom et al., 2007; Lewcock et al., 2007; Culican et al., 2009; Hendricks and Jesuthasan, 2009). The mechanisms underlying these axonal defects are not well understood at the cellular and molecular level, and it is not clear whether the Wnd/DLK pathway is responsible for the deficits.

In this study, we investigate the role of the Drosophila PHR, Highwire, in $\mathrm{MB}$ axon development. Mutations in highwire disrupt $\mathrm{MB}$ lobe morphology. Single-axon analysis demonstrates that mutant axons bifurcate normally, but the sister branches fail to segregate and instead project together into the same lobe. This guidance defect is suppressed by mutations in Wnd/DLK, demonstrating the involvement of this MAPKKK pathway. We show that Highwire acts via a non-cell-autonomous mechanism and find that Highwire interacts with a Plexin A-dependent signaling pathway to control axon development. Together, our study demonstrates that Highwire regulates guidance of sister branches in the $\mathrm{MB}$ via a non-cell-autonomous mechanism that may involve Plexin A signaling.

\section{Materials and Methods}

Fly stocks. Drosophila melanogaster were raised on standard fly food at $25^{\circ} \mathrm{C}$. Canton S was used as wild-type (WT) flies if not specified otherwise. highwire mutant alleles, $h i w^{N D 8}$ and $h i w^{\Delta N}$, and the highwire transgenes, UAS-hiw, UAS-GFP-hiw and UAS-hiw $\triangle R I N G$, have been described previously (Wan et al., 2000; Wu et al., 2005). $w n d^{l}$ and $w n d^{3}$ 
mutant alleles were described by Collins et al. (2006). UAS-mCD8-GFP was used to label cellular membranes (Lee and Luo, 1999). The following enhancer trap and mutant lines were obtained from the Bloomington Stock Center: OK107-Gal4, 30y-Gal4, plexA ${ }^{\text {YY16548 }}$, plexB ${ }^{\text {KG878 }}$, Sema$1 a^{K 13702}$ slit $^{2}$, robo3 $^{\text {MB495 }}$, robo2 $^{\text {EY20629 }}$, Dscam $^{1}$, Dscam $^{P Z 5518}$, Pak $^{6}$, dock $^{P Z 4723}$, and Ephrin ${ }^{K G 118}$. The NP enhancer trap lines NP2748-Gal4 and NP7175-Gal4 were obtained from the Drosophila Genetic Resource Center (Kyoto, Japan). UAS-plexA RNAi (VDRC107004) and UASSema-1a RNAi (VDRC104505) transgenic lines were obtained from the Vienna Drosophila RNAi Center (Vienna, Austria). The Sema- $5 c^{B G 01245}$ mutant was a gift from Dr. R. Anholt (North Carolina State University, Raleigh, NC) (Rollmann et al., 2007). The stocks used for the mosaic analysis with a repressible cell marker (MARCM) experiments were obtained from the Bloomington Stock Center and include $h s-F L P 22$, FRTG13,tub-Gal80, hs-FLP,tub-Gal80,FRT19A; UAS-mCD8-GFP, and FRT19A. Male flies were used for the analyses except for generating hiw $^{\Delta N}$ mosaic clones.

Single-cell analyses. Mitotic single cell clones were generated in the MB by taking the MARCM approach as described by Wu and Luo (2006). To produce GFP-labeled clones in WT and hiw mutant MBs, hs-FLP; FRTG13,tub-Gal80/CyO and hiw ${ }^{\Delta N}, h s-F L P ; F R T G 13, t u b-G a l 80 / C y O$ flies were each crossed to +/y; FRTG13,UAS-mCD8-GFP; OK107-Gal4. The MARCM-ready animals were exposed to heat shock at $37^{\circ} \mathrm{C}$ to induce flippase recombinase (FLP)-mediated mitotic recombination and, thus, clonal expression of mCD8-GFP. Heat shock was given at the pupal stage for $20 \mathrm{~min}$ for optimal generation of single $\alpha / \beta$ cell clones. For the cell-autonomy studies, hs-FLP,tub-Gal80,FRT19A/y; UAS$m C D 8-G F P$ flies were crossed to either FRT19A; OK107-Gal4 or $h_{i w}{ }^{\Delta N}$,FRT19A; OK107-Gal4. Heat shock was given to generate mosaic clones as explained above. MARCM clones were immunolabeled with anti-GFP antibodies (Invitrogen), whereas anti-Fasciclin II (Fas II) staining was used to define the $\alpha / \beta$ lobe area. MBs with multiple labeled neurons were excluded from the morphological analysis of single axons because of the difficulty in tracing each axon.

Immunohistochemistry. Brains from adults or pupae were dissected in cold PBS with $0.1 \%$ Triton X-100 (PBS-T) and fixed in $4 \%$ paraformaldehyde. After washes with PBS-T, brains were blocked in 5\% NGS in PBS-T for $3 \mathrm{~h}$ at room temperature and incubated with primary antibodies diluted in PBS containing 5\% NGS and 0.7\% Triton $\mathrm{X}-100$ for $2-3 \mathrm{~d}$ at $4^{\circ} \mathrm{C}$. Brains were then washed three times with PBS-T, followed by incubation with secondary antibodies in 5\% NGS in PBS-T for $3 \mathrm{~h}$ at room temperature, three washes in PBS-T, equilibrium in $70 \%$ glycerol in PBS, and mounting in VectaShield (Vector Laboratories). For staining larval MBs, fillet preparations were made from third-instar larvae, and the larvae were subjected to the staining procedure described above.

The following primary antibodies were used: mouse anti-Fas II at 1:5 (clone 1D4), rabbit anti-Wnd at 1:1000 (Collins et al., 2006), and rabbit anti-GFP conjugated to Alexa Fluor-488 at 1:200 (Invitrogen). Cy3conjugated anti-mouse or rabbit secondary antibodies were purchased from Jackson ImmunoResearch and used at 1:1000.

Confocal imaging and statistical analysis for $M B$ phenotypes. Samples were imaged with a Nikon D-Eclipse C1 confocal microscope using $40 \times$ or $60 \times$ oil objective. Images shown are $z$-projections of confocal stacks acquired from serial laser scanning except for the Wnd immunostaining, which is presented as single confocal sections. To assess the lobe width, samples were blinded to genotype, each MB was categorized into "normal" and "defective" classes, and the phenotypic severity in each group was presented as the percentages of MBs that belong to the defective class. Statistical significance was assessed by either the Fisher's exact test or the $\chi^{2}$ test depending on the sample sizes. For genetic interaction studies with the plexin A (plexA) mutation, $\mathrm{MB}$ defects were categorized into three classes to sensitize the analysis: normal, medium, and strong as described in Results. Samples were scored under a fluorescence microscope, and the confidence of the scoring method was confirmed by performing analysis of skewness. To evaluate the skewness between $\alpha$ and $\beta$ lobes, widths of the $\alpha$ and $\beta$ lobes were measured from confocal image of each MB (NIH ImageJ) and applied to the following equation: skew- ness $=\frac{|a-b|}{a+b}$, when $a$ and $b$ are widths of $\alpha$ and $\beta$ lobes, respectively. Greater skewness represents more difference between the widths of the two lobes and, thus, a stronger defect.

$M B$ cell counting. Number of Kenyon cell bodies was estimated by counting the cells labeled with OK107-Gal4-driven mCD8-GFP. The cell counter plug-in of NIH ImageJ was used to count cells in every fifth images in the confocal stacks that were taken at $0.5 \mu \mathrm{m}$ intervals.

\section{Results}

\section{Highwire is required for normal MB structures in adult brains}

During development, MB neurons, called Kenyon cells, undergo an ordered differentiation process into three types of neurons: $\alpha / \beta$ neurons, $\alpha^{\prime} / \beta^{\prime}$ neurons, and $\gamma$ neurons (Lee et al., 1999). The cell bodies of these MB neurons are located in the posterior of the brain and project their axons to the anterior region through an axon tract called the peduncle (Fig. $1 A$ ). At the anterior end of the peduncle, each axon bifurcates, and the resulting two branches are segregated into two perpendicular lobes. Consequently, axon branches from $\alpha / \beta$ neurons generate an $\alpha$ (dorsal) lobe and a $\beta$ (medial) lobe, whereas those from $\alpha^{\prime} / \beta^{\prime}$ neurons form the $\alpha^{\prime}$ (dorsal) lobe and $\beta^{\prime}$ (medial) lobe. Although $\gamma$ neurons initially generate dorsal projections as well as medial projections, the larval dorsal axons are pruned during metamorphosis, leaving only a medial $\gamma$ lobe. Antibodies against an axon fascicle component, Fas II, label the $\alpha$ and $\beta$ lobes strongly and $\gamma$ lobe weakly in WT adult brains (Fig. $1 A, B$ ). The WT $\alpha$ and $\beta$ axonal lobes show similar widths, indicating the presence of similar number of axons in the two lobes. In hiw mutants, however, this stereotyped morphology of MB lobes is disrupted. Fas II immunostaining of brains from the highwire deletion mutant $h i w^{\Delta N}$ (Fig. $1 B$ ) demonstrates that one of the two lobes is often much thinner than the other, and occasionally one lobe is nearly absent (Fig. $1 \mathrm{~B}$, asterisk). This unequal distribution of the axonal projections between the $\alpha$ and $\beta$ lobes was observed in $86 \%$ of the hiw ${ }^{\Delta N}$ MBs $(n=$ $70)$, whereas all the examined WT MBs were normal $(n=85)$. This phenotype is also present in a second, genetically independent highwire allele, $h_{i w^{N D}}$, an EMS allele with a premature stop codon (see Fig. 7), but the phenotype is less severe (66\% penetrance, $n=143$ ). The hypomorphic nature of hiw ${ }^{N D 8}$ was not observed in our previous studies of synapse development but is consistent with the read-through of the stop codon and expression of some full-length Highwire protein in this nonsense allele (Wu et al., 2005). We used the strong $h i w^{\Delta N}$ allele for a more complete phenotypic characterization of the highwire mutant MB. Thicker $\alpha$ lobes with thinner $\beta$ lobes are most commonly observed in highwire mutants, but MBs with thicker $\beta$ lobes are also present. Interestingly, we occasionally observe a thinner $\beta$ lobe on one side of the brain and a thicker $\beta$ lobe on the other side of the brain, suggesting that the underlying defect is independently determined in the two brain hemispheres (Fig. $1 B$ ). In addition to the abnormal thickness of the $\alpha$ and $\beta$ lobes, there is a second phenotype in the highwire mutant $\mathrm{MB}$, which often has a shortened $\alpha$ lobe ( hiw $^{\Delta N}, 39 \%$ penetrance, $n=70$; Fig. $1 B$, arrowhead). The aberrant Fas II staining in the $\alpha$ and $\beta$ lobes could represent a change in Fas II expression/localization or a change in the anatomy of the axons. To distinguish between these possibilities, we labeled the axons by expressing a membraneassociated mCD8-GFP using the pan-Kenyon cell driver OK107Gal4. The phenotype is readily apparent with mCD8-GFP (Fig. 1C), demonstrating that there is a defect in axon morphology in the highwire mutants. In addition, the number of GFP-labeled Kenyon cells 
A

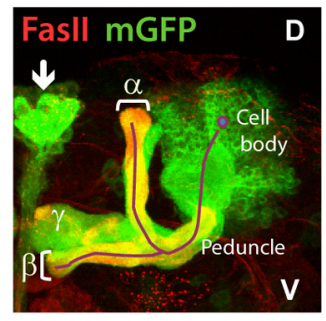

C
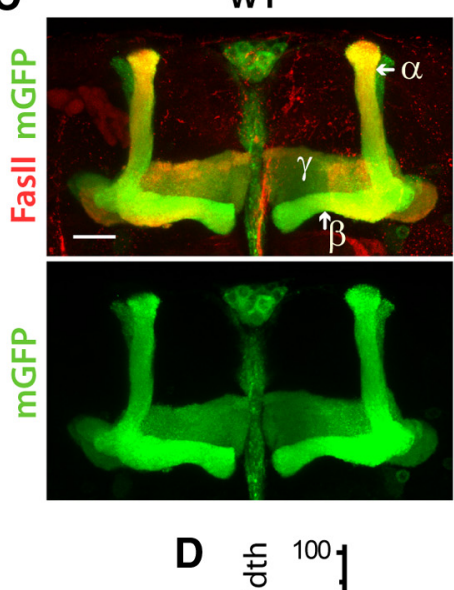

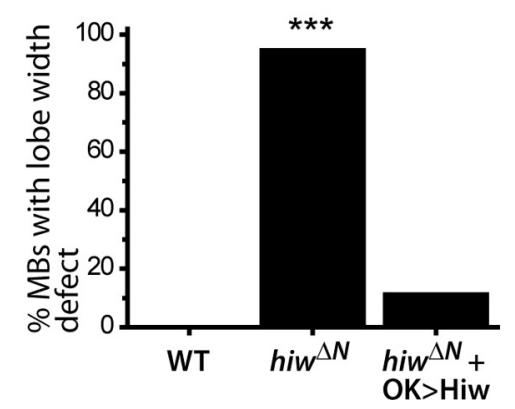

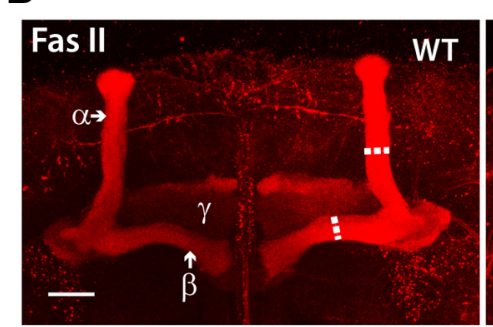

$\operatorname{hiw}^{\Delta N}$
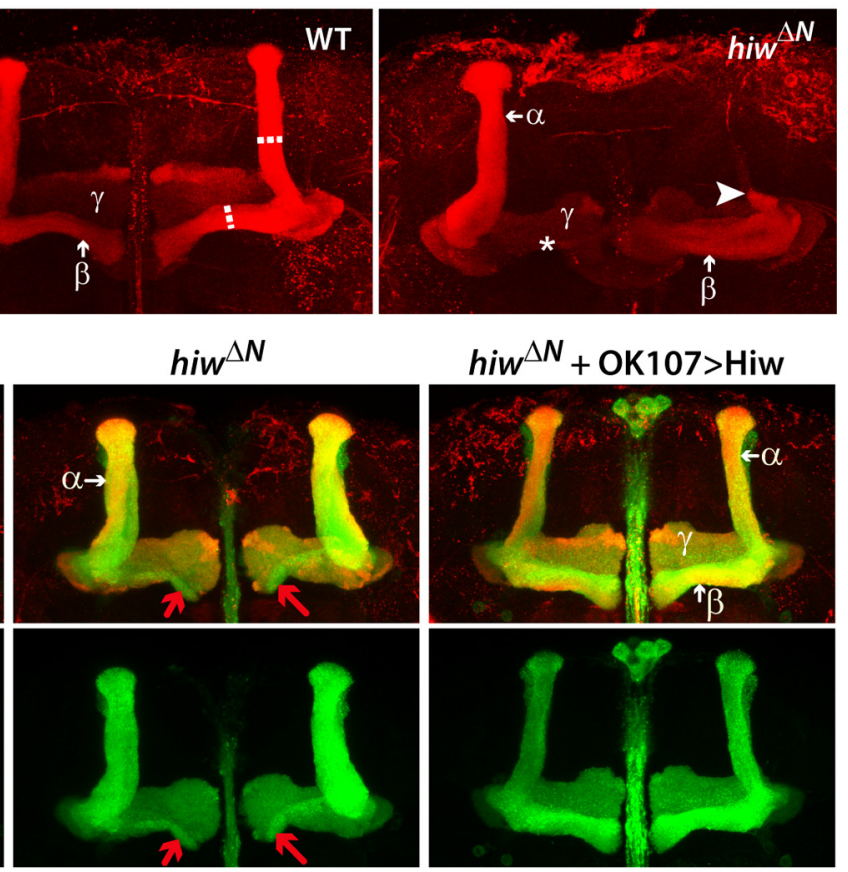

hiw $^{\Delta N}+$ OK107> Hiw

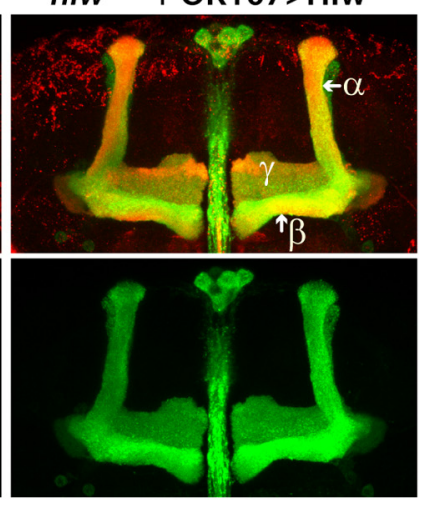

E 30y>GFP-Hiw

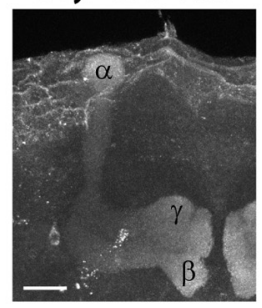

Figure 1. Highwire is required for normal structures of adult MB. A, Adult MB visualized with anti-Fas II immunolabeling (red) and mCD8 -GFP (green) driven by a pan-MB neuronal driver, OK107-Gal4. Fas II is expressed strongly in the $\alpha / \beta$ lobes and weakly in the $\gamma$ lobe. Schematic of an $\alpha / \beta$ axon is shown in purple line. Arrow indicates cells in the pars intercerebralis. D, Dorsal; $V$, ventral. $\boldsymbol{B}$, Images of WT and highwire mutant (hiw ${ }^{\Delta N}$ ) MB in adult brains. Fas II labeling shows similar widths of $\alpha$ and $\beta$ lobes (dashed lines) in WT, whereas highwire mutant MBs display loss (asterisk) or shortening (arrowhead) of either lobe. $C$, The defects in the highwire $\alpha$ and $\beta$ lobes are rescued by transgenic expression of highwire in the MB neurons. MBs from WT (+/y;

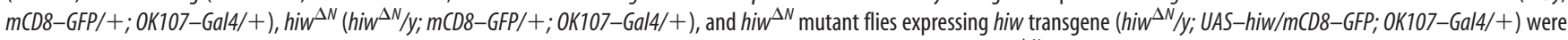
visualized by mCD8 -GFP driven by 0K107-Gal4 and immunostaining for Fas II. Red arrows indicate thinning of $\beta$ lobes in hiw ${ }^{\Delta N}$. D, Results from $C$ were quantified for the percentage of MBs with the width defect in $\alpha / \beta$ lobes. WT, $n=20 ;$ hiw $^{\Delta N}, n=34 ;$ hiw $^{\Delta N}+0$ K107 $>$ Hiw, $n=28 ;{ }^{* * *} p \ll 0.001$ versus WT and hiw ${ }^{\Delta N}+0$ OK107 $>$ Hiw. E, Transgenic expression of GFP-hiw by a pan-MB neuronal driver, 30y-Gal4. Brains from 30y-Gal4/UAS-GFP-hiw were stained with anti-GFP antibodies to show the localization of the transgenic proteins. Scale bars, $20 \mu \mathrm{m}$.

in highwire is comparable with that in WT $\left(2246 \pm 57\right.$; iw $^{\Delta N}$, $2291 \pm 142 ; n=6$ for each genotype; $p>0.7)$, suggesting that cell proliferation is not significantly affected by the loss of Highwire function.

To test whether the loss of Highwire is responsible for the observed $\mathrm{MB}$ axonal phenotypes, we performed rescue experiments by expressing the highwire transgene with OK107-Gal4 in the hiw ${ }^{\Delta N}$ background (Fig. 1C). The transgenic expression of highwire results in a restoration of normal MB thickness in $89 \%$ of examined MBs (Fig. $1 D ; p \ll 0.001$ ). The defect of shortened $\alpha$ lobe is also rescued ( $h i w^{\Delta N}, 54 \%, n=24 ; h i w^{\Delta N}+$ OK107 $>$ highwire, 4\%, $n=28$; $p \ll 0.001$ ), confirming that Highwire expression within the Kenyon cell population is necessary for the organization of the MB. We then tested the requirement of the Highwire E3 ligase activity for $\mathrm{MB}$ development by performing a rescue experiment with a highwire transgene in which the key cysteine residues in the RING finger domain are mutated (Wu et al., 2005). Expression of this transgene, hiw $\Delta R I N G$, essentially rescues the $\alpha$ and $\beta$ lobe width defect in the hiw ${ }^{\Delta N}$ background $\left(h_{i w}{ }^{\Delta N}, 94 \%, n=34 ;\right.$ hiw $^{\Delta N}+$ OK107 $>$ hiw $\Delta$ RING $, 25 \%, n=8$; $p<0.001$ ), whereas the shortened $\alpha$ lobe phenotype is not rescued $\left(\right.$ hiw $^{\Delta N}+$ OK107 $>$ hiw $\Delta$ RING, 63\%, $\left.n=8 ; p=1\right)$. This result suggests that a WT ligase domain is necessary for the normal Highwire function but RING mutant still preserves some functionality, although it is possible that the expression level of the hiw $\Delta$ RING transgene may not be sufficient for complete rescue.

Although we do not have an antibody to Highwire that works for tissue staining, we did assess Highwire localization by expressing a functional GFP-tagged highwire transgene (Wu et al., 2005) with a pan-MB driver, 30y-Gal4. Hiw-GFP is detected in axons of all MB lobes, $\alpha / \beta, \alpha^{\prime} / \beta^{\prime}$, and $\gamma$, so it could function within the axonal compartment (Fig. $1 E$ ).

\section{The highwire MB phenotypes are developmental defects}

The morphology of MBs can be disrupted by developmental and/or degenerative defects in axons (Rallis et al., 2010). Devel- 
1 day APF
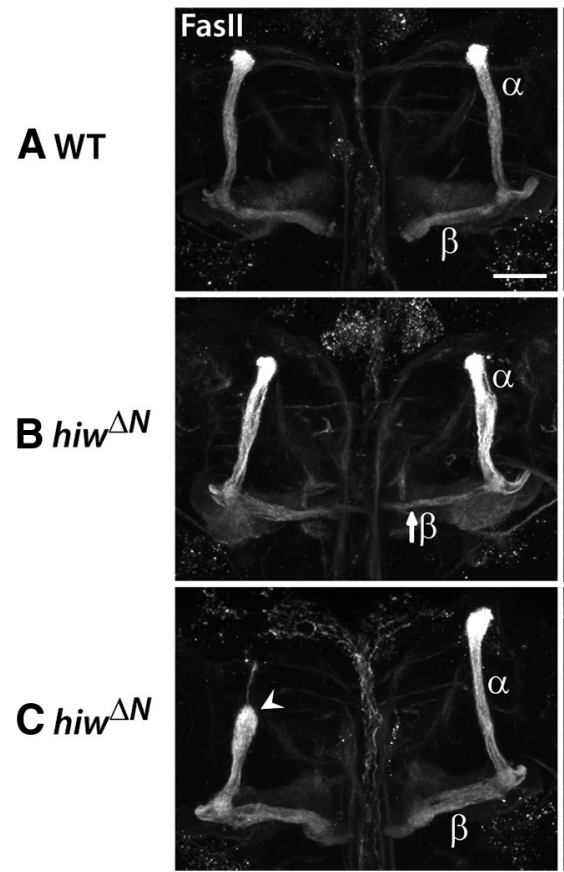

2 day APF
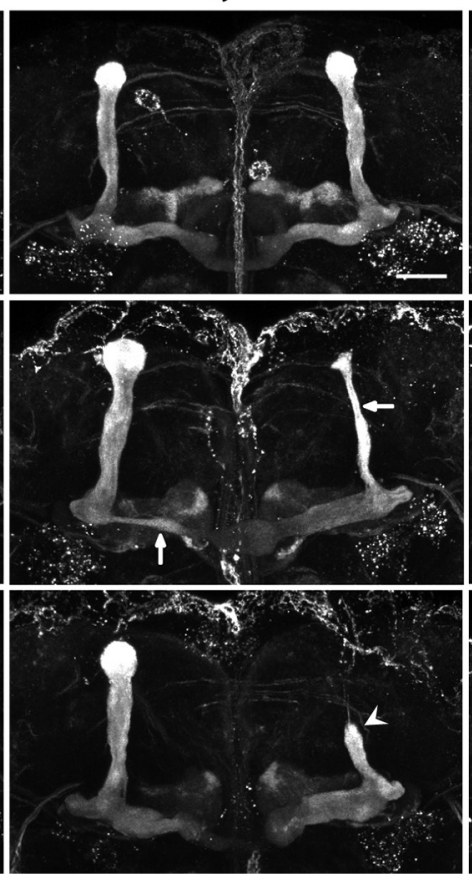

3 day APF

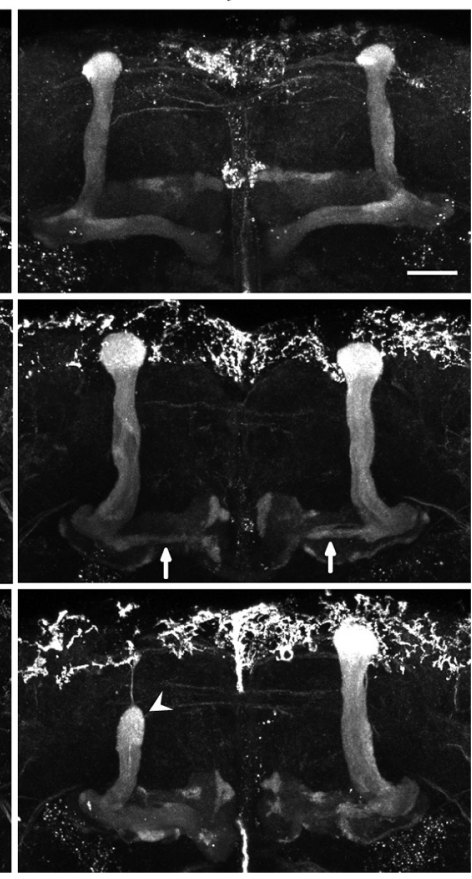

Figure 2. Loss of highwireaffects development of $\alpha / \beta$ lobes. Immunolabeling for Fas II stains $\alpha / \beta$ lobes from WT $(\boldsymbol{A})$ and highwiremutant ( $\boldsymbol{B}, \boldsymbol{C})$ pupae at indicated times APF. $\boldsymbol{A}$, WT MBs display comparable widths of $\alpha$ and $\beta$ lobes throughout pupal development. $\boldsymbol{B}, \boldsymbol{C}$, Thinning of either lobe (arrows in $\boldsymbol{B}$ ) and shortening of $\alpha$ lobe (arrowheads in $\boldsymbol{C}$ ) were observed in hiw ${ }^{\Delta N}$ as early as $1 \mathrm{~d}$ APF. Scale bars, $20 \mu \mathrm{m}$.

opmental abnormalities in axonal growth, branching, or guidance or degeneration leading to axon loss could result in short or thin MB lobes observed in highwire mutant brains. To differentiate between these possibilities, we analyzed mutant brains at earlier developmental stages. As shown by Fas II labeling, the defect in $\alpha$ and $\beta$ lobe widths is observed in the pupal MB of hiw ${ }^{\Delta N}$ mutants at the time when $\alpha / \beta$ axons are still developing. The WT MBs at one day after puparium formation (APF) have $\alpha$ and $\beta$ lobes with similar width, although they are thinner than those found in adults (Fig. $2 \mathrm{~A}$ ). In contrast, the defect in lobe width is readily observed in the $h i w^{\Delta N}$ brains (54\% penetrance, $n=13$; Fig. $2 B$ ) and persists throughout pupal development (62\% penetrance at $3 \mathrm{~d}$ APF, $n=16)$. Moreover, the short $\alpha$ lobe phenotype is also observed at the pupal stages (Fig. 2C, arrowheads) and does not become exacerbated in adult flies (2 and 6 weeks after eclosion; data not shown), arguing against age-related retraction of the axons. These data indicate that the $\alpha$ and $\beta$ lobe phenotypes of highwire mutants are consequences of developmental defects.

highwire selectively disrupts $\alpha / \beta$ lobes

$\alpha^{\prime} / \beta^{\prime}$ neurons develop dorsal $\left(\alpha^{\prime}\right)$ and medial $\left(\beta^{\prime}\right)$ lobes like $\alpha / \beta$ neurons. We next asked whether Highwire is also required for $\alpha^{\prime}$ and $\beta^{\prime}$ lobe development as it is for $\alpha$ and $\beta$ lobe formation. Because $\alpha^{\prime} / \beta^{\prime}$ lobes are not labeled by Fas II staining and the $\gamma$ lobe is only weakly labeled in pupae and adults, we used mCD8GFP expression using Gal4 drivers to examine their morphology. The development of $\alpha^{\prime} / \beta^{\prime}$ axons takes place during the thirdinstar larval stage, which is later than the development of $\gamma$ lobe (first-instar larva) but earlier than the development of $\alpha / \beta$ lobes (pupa). Therefore, by analyzing brains of the third-instar larvae, we could examine the structure of the $\alpha^{\prime} / \beta^{\prime}$ and $\gamma$ lobes without interference from the $\alpha / \beta$ lobe projections. In the WT larval brains, both $\alpha^{\prime} / \beta^{\prime}$ and $\gamma$ neurons comprise the dorsal and the medial lobe as visualized with $\mathrm{mCD} 8$-GEP expression driven by
OK107-Gal4 (Fig. 3A). Interestingly, MB structure in the hiw ${ }^{\Delta N}$ mutant larvae appears normal in the width and length of dorsal and medial lobes $(100 \%, n=20)$, indicating that the axons of $\alpha^{\prime} / \beta^{\prime}$ and $\gamma$ neurons are not affected at the larval stage. We further analyzed the mutant $\alpha^{\prime} / \beta^{\prime}$ and $\gamma$ lobes at later developmental stages. In contrast to the dramatically impaired $\alpha / \beta$ lobes $(82 \%, n=22), \alpha^{\prime} / \beta^{\prime}$ and $\gamma$ lobes in the late pupal $h i w^{\Delta N}$ mutant maintain essentially normal structures (100\%, $n=10$; Fig. $3 B)$. The morphology (length and width) of a mutant $\alpha^{\prime}$ lobe is comparable with that in WT even when the neighboring $\alpha$ lobe in the same MB is noticeably short (Fig. $3 B$, arrowhead). $\alpha^{\prime} / \beta^{\prime}$ and $\gamma$ lobes remain intact in the adult stage when the $\alpha^{\prime} / \beta^{\prime}$ neuronal driver NP2748-Gal4 (Tanaka et al., 2008) was used to express mCD8-GFP exclusively in the $\alpha^{\prime} / \beta^{\prime}$ lobes (Fig. $3 C$ ). The GFPlabeled $\alpha^{\prime} / \beta^{\prime}$ lobes and the Fas II-labeled $\gamma$ lobe display normal width and length $(96 \%, n=26)$, even when the $\alpha / \beta$ lobes are impaired within the same MB. Collectively, these data show that Highwire function is not essential for the organization of $\alpha^{\prime} / \beta^{\prime}$ and $\gamma$ axonal lobes and that the loss of Highwire preferentially affects the development of $\alpha / \beta$ projections.

\section{Highwire controls guidance of sister axon branches in $\boldsymbol{\alpha} / \boldsymbol{\beta}$ neurons}

Developing axons of $\alpha / \beta$ neurons normally bifurcate at the base of the lobes, and the resulting sister branches subsequently extend in diverging directions: one to the $\alpha$ lobe and the other to the $\beta$ lobe. At the cellular level, the $\alpha / \beta$ width phenotype we find in highwire mutants could be attributable to two possible defects: (1) the highwire axon does not bifurcate and grows to one lobe, reducing the number of axonal projections in the other lobe, or (2) sister branches are formed but both project to a single lobe (Fig. 4A). Therefore, we next examined whether Highwire is required for the branch generation and/or for the guidance of branches. To visualize fewer axons in the $\alpha$ and $\beta$ lobes, we used a driver expressed in the subset of neurons that comprise the core 
A
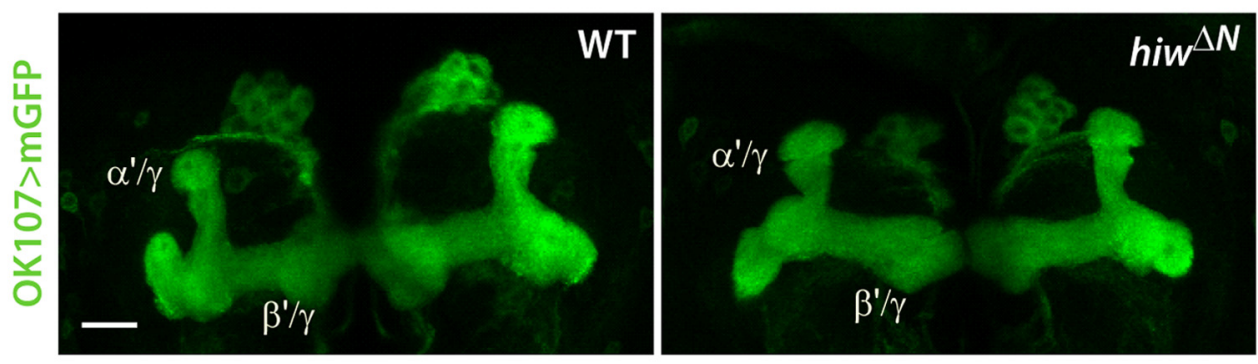

B
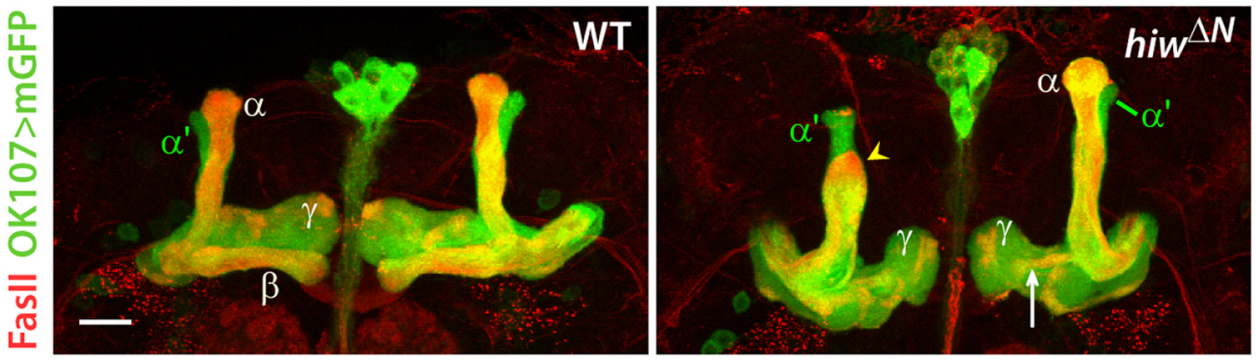

C

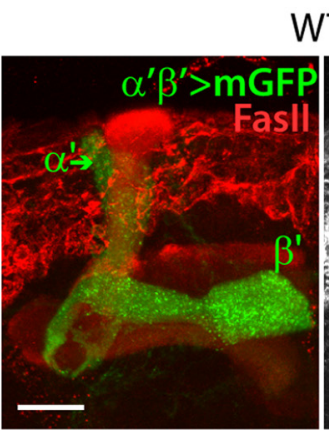

WT
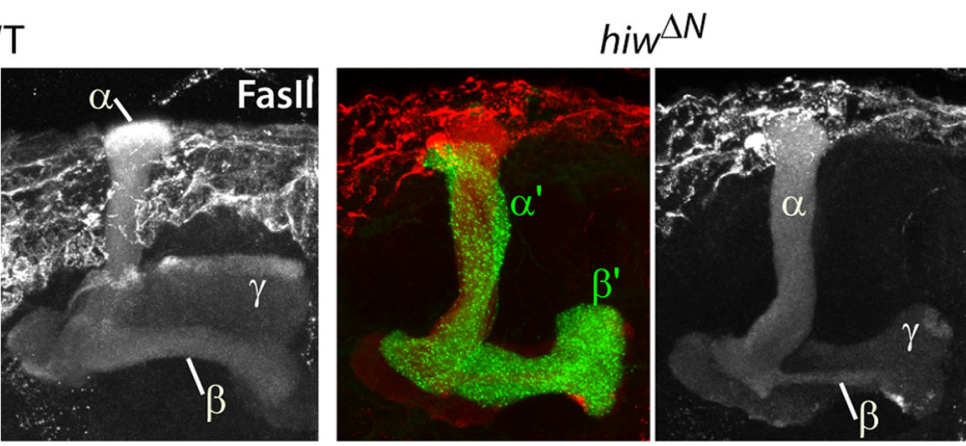

Figure 3. $\alpha / \beta$ lobes are preferentially impaired in highwire mutant MB. $\boldsymbol{A}$, Structures of $\gamma, \alpha^{\prime}$, and $\beta^{\prime}$ lobes are comparable between WT and hiw ${ }^{\Delta N}$ third-instar larvae. The larval MBs were labeled with mCD8 -GFP expression by OK107-Gal4. B, $\alpha$ and $\beta$ lobes in $h i w^{\Delta N}$ mutant pupae display thinning (arrow) or shortening (arrowhead) of the lobes, whereas $\alpha^{\prime}, \beta^{\prime}$, and $\gamma$ lobes are largely normal. mCD8 -GFP (green) driven by 0K107-Gal4 labels all pupal lobes $\left(\gamma, \alpha^{\prime}, \beta^{\prime}, \alpha\right.$, and $\beta$ ), whereas only $\alpha$ and $\beta$ lobes are strongly stained for Fas II (red). Genotype in $\boldsymbol{A}$ and $\boldsymbol{B}$ : WT

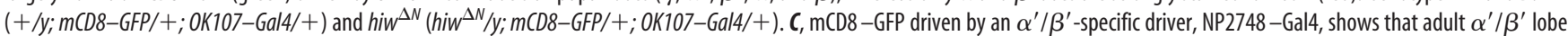
structures in hiw ${ }^{\Delta N}$ (hiw ${ }^{\Delta N}, N P 2748-G a l 4 / y ; m$ CD8-GFP/+) brains are comparable with those in WT (NP2748-Gal4/y; mCD8-GFP/+). Scale bars, $20 \mu \mathrm{m}$.

region of $\alpha$ and $\beta$ lobes (NP7175-Gal4) (Tanaka et al., 2008). In WT, mCD8-GFP expression by the driver shows the presence of a core axon fascicle in each of the two lobes. However, in the $\alpha$ and $\beta$ lobes of hiw ${ }^{\Delta N}$ mutants, there are two separate core axon fascicles that aberrantly project into the same thick lobe (Fig. $4 B$ ). To confirm that the separate core fascicles reflect the behavior of sister branches from an individual neuron, we labeled single cells with GFP in either WT or hiw ${ }^{\Delta N}$ background (Wu and Luo, 2006). In our experiments GFP-positive clone formation was induced at the pupal stage to preferentially mark $\alpha / \beta$ neurons that undergo cell division in pupae. In WT brains, the sister branches from a single $\alpha / \beta$ neuron project into the $\alpha$ and $\beta$ lobes. In contrast, labeled single cells in the hiw ${ }^{\Delta N}$ background extend axons through the peduncle, bifurcate normally, but fail to project the sister axons in divergent directions (94\%, $n=16$; Fig. $4 C$ ). Thus, single-axon labeling demonstrates that Highwire function is not essential for axonal branch formation but is required for the guidance of axons to their correct target.

Highwire controls $\mathrm{MB}$ formation by restricting the level of the MAPKKK Wallenda

Highwire controls synaptic growth at the fly neuromuscular junction (NMJ) by restricting the protein level of Wnd, an
MAPKKK homologous to vertebrate DLK (Collins et al., 2006). In highwire mutants, the Wnd level at the NMJ is notably elevated. The increased activity of Wnd is responsible for the synaptic overgrowth phenotype in highwire as demonstrated by genetic suppression experiments in which the defect is suppressed by loss-of-function mutations in wnd. Therefore, we asked whether Highwire governs MB axon development via this MAPKKK pathway. First, we checked whether Highwire regulates the protein level of Wnd in $\mathrm{MB}$ by performing immunostaining with anti-Wnd antibodies (Collins et al., 2006). In WT, no prominent staining is detected from either larval or pupal MB, except some immunoreactivity at the center of the peduncles. In contrast, Wnd is easily detected in the hiw ${ }^{\Delta N} \mathrm{MBs}$ at both larval and pupal stages (Fig. $5 A$, pupal brains are not shown). Hence, Highwire normally restricts Wnd levels in the MB. Wnd levels in hiw ${ }^{\Delta N}$ are particularly elevated in the lobes and the peduncle and also slightly increased in the cell bodies (lobe, $4.7 \pm 0.7$-fold increase, $n=6, p<0.001$; peduncle, $4.0 \pm 0.3$-fold increase, $n=5, p \ll$ 0.001 ; cell body, $1.5 \pm 0.1$-fold increase, $n=6, p<0.05)$. We hypothesized that this increase in Wnd levels is functionally relevant for the axonal defects observed in the highwire MB. Consistent with this view, loss of whd suppresses the hiw ${ }^{\Delta N} \mathrm{MB}$ phenotype (Fig. $5 B, C ; p<0.001$ ). Mutations in $w n d$ alone do not 
A

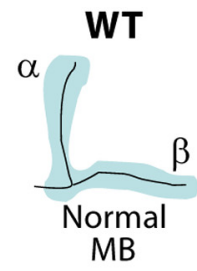

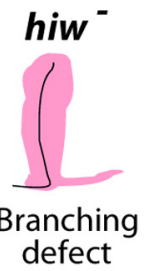

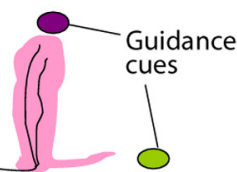

Guidance defect

B
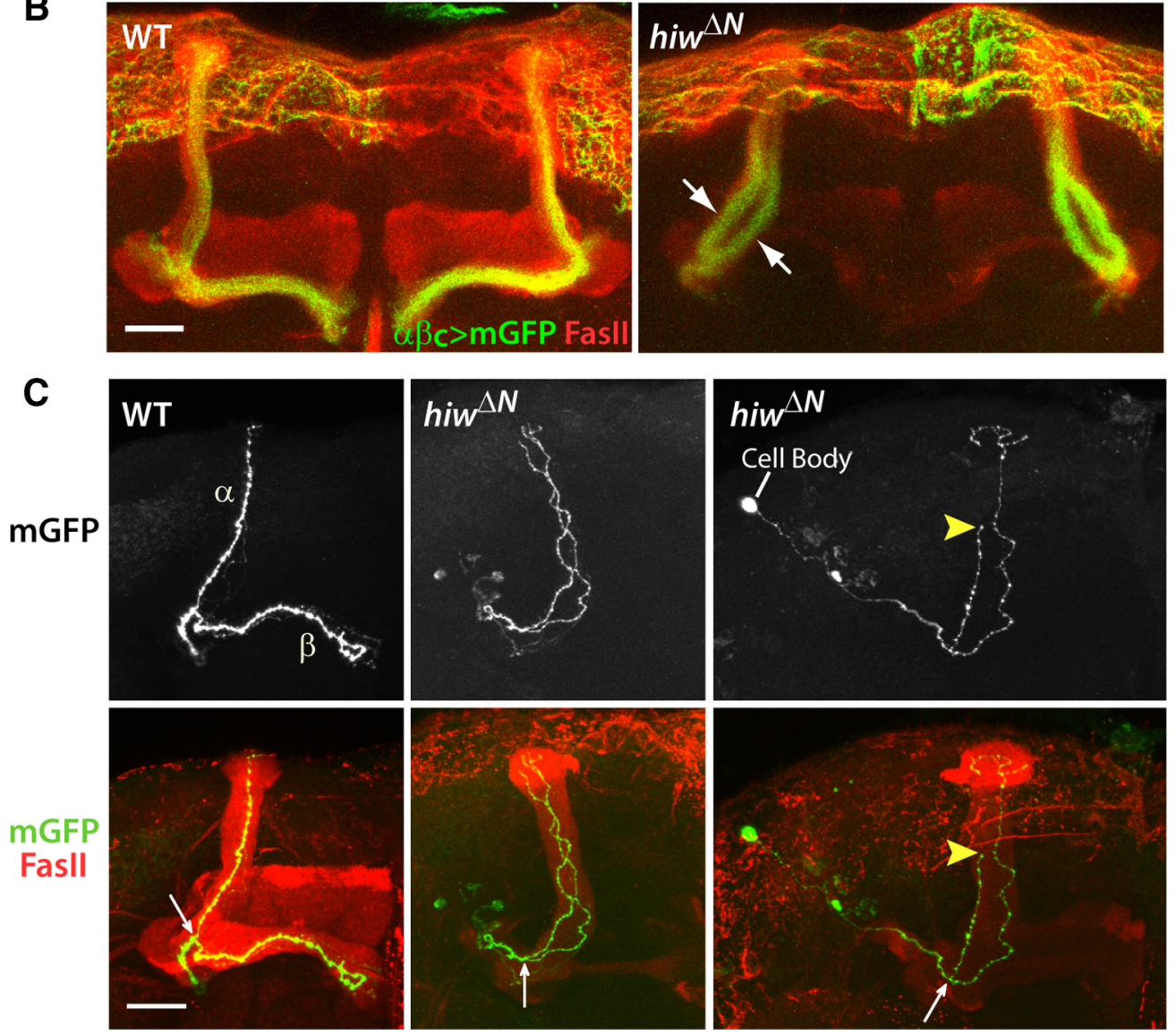

Figure 4. Sister branches of $\alpha / \beta$ axons fail to segregate into different lobes in the highwire mutant. $A$, A model for the highwire MB phenotype at the single-cell level. Thinning of an MB lobe can be attributable to the failure in branch formation or the defect in the guidance of sister axon branches. $B$, Core axons in $\alpha / \beta$ lobes form two distinct fascicles in WT and hiw ${ }^{\Delta N}$ MB, but in the hiw $^{\Delta N}$ mutant, the fascicles abnormally extend into the same lobe (arrow). mCD8 -GFP driven by NP7175-Gal4 marks central axons in the $\alpha$ and $\beta$ lobes. MBs in WT (NP7175-Gal4/y; $m$ CD8-GFP/+) and hiw $^{\Delta N}$ (hiw $\left.{ }^{\Delta N}, N P 7175-G a l 4 / y ; m C D 8-G F P /+\right)$ flies were stained with antibodies to Fas II (red) and GFP (green). C, Single-cell labeling with GFP in WT and hiw ${ }^{\Delta N}$ adult MBs. In hiw ${ }^{\Delta N}$ mutant background, $\alpha / \beta$ axons normally bifurcate at the branching point (arrow) but aberrantly project to the same lobe. Arrowhead indicates an abnormally short axon in a hiw ${ }^{\Delta N} M B$. WT (hs-FLP/y; FRTG13,tub-Gal80/FRTG13, mCD8-GFP; OK107-Gal4/+) and iw $^{\Delta N}$ (hiw ${ }^{\Delta N}$, hs-FLP/y; FRTG13,tub-Gal80/FRTG13, mCD8-GFP; 0K107-Gal4/+) brains were immunostained for Fas II and GFP to label the single-cell clones. Scale bars, $20 \mu \mathrm{m}$.

impair the structure of $\alpha$ and $\beta$ lobes. Together, these results demonstrate that Highwire regulates axon development in MB by downregulating the Wnd protein.

\section{Highwire affects $\boldsymbol{\alpha} / \boldsymbol{\beta}$ axon formation via a non-cell-autonomous mechanism}

$\mathrm{MB}$ axons extend as organized fascicles through the peduncle and the lobes. Within the bundled structure, projection of an axon depends on the trajectories of surrounding axons attributable to interactions between axons. For instance, mutations in Dscam disrupt overall organization of $\alpha$ and $\beta$ lobes via a non-cellautonomous mechanism that controls axon guidance (Wang et al., 2002). We thought that Highwire might also play a non-cellautonomous role in the MB axon development for three reasons. (1) The great difference in the widths of $\alpha$ and $\beta$ lobes implies that the guidance choice of individual axon is not purely random in highwire; if axons made independent decisions, the two lobes would contain similar numbers of projections by chance. (2) The identity of the thickened lobe varies across brain hemispheres-in the same animal, the $\alpha$ lobe may be thick on the right whereas the $\beta$ lobe is thick on the left. This implies that the defect is not in response to a localized guidance cue that promotes either $\alpha$ or $\beta$ lobe formation. Instead, the observed phenotype is consistent with losing non-cell-autonomous factors affecting the choice of most $\alpha / \beta$ neurons, such as pioneer axons that subsequently affect the development of followers. (3) We observed that Wnd levels in the highwire mutant MB are elevated even in the $\alpha^{\prime} / \beta^{\prime}$ and $\gamma$ lobes that do not manifest notable phenotypes, demonstrating a role for Highwire in those lobes. In fact, these earlierborn $\alpha^{\prime} / \beta^{\prime}$ axons may act as pioneering projections that guide later-born $\alpha / \beta$ axons (Wang et al., 2002). Therefore, we tested the possibility that Highwire influences $\alpha / \beta$ lobe formation by a non- 
A
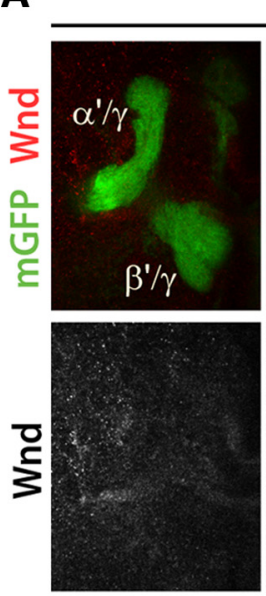

WT
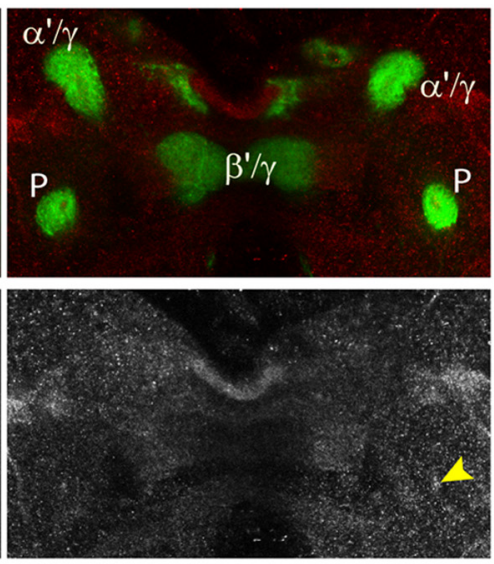
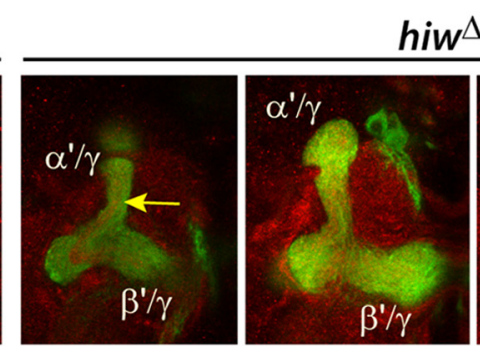

$\operatorname{hiw}^{\Delta N}$
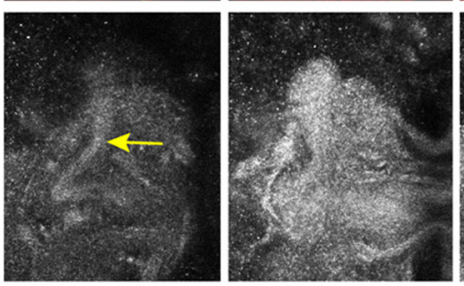
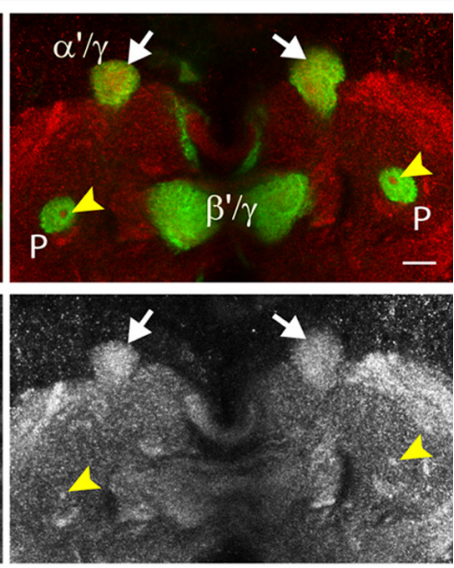

\section{B Fasil}
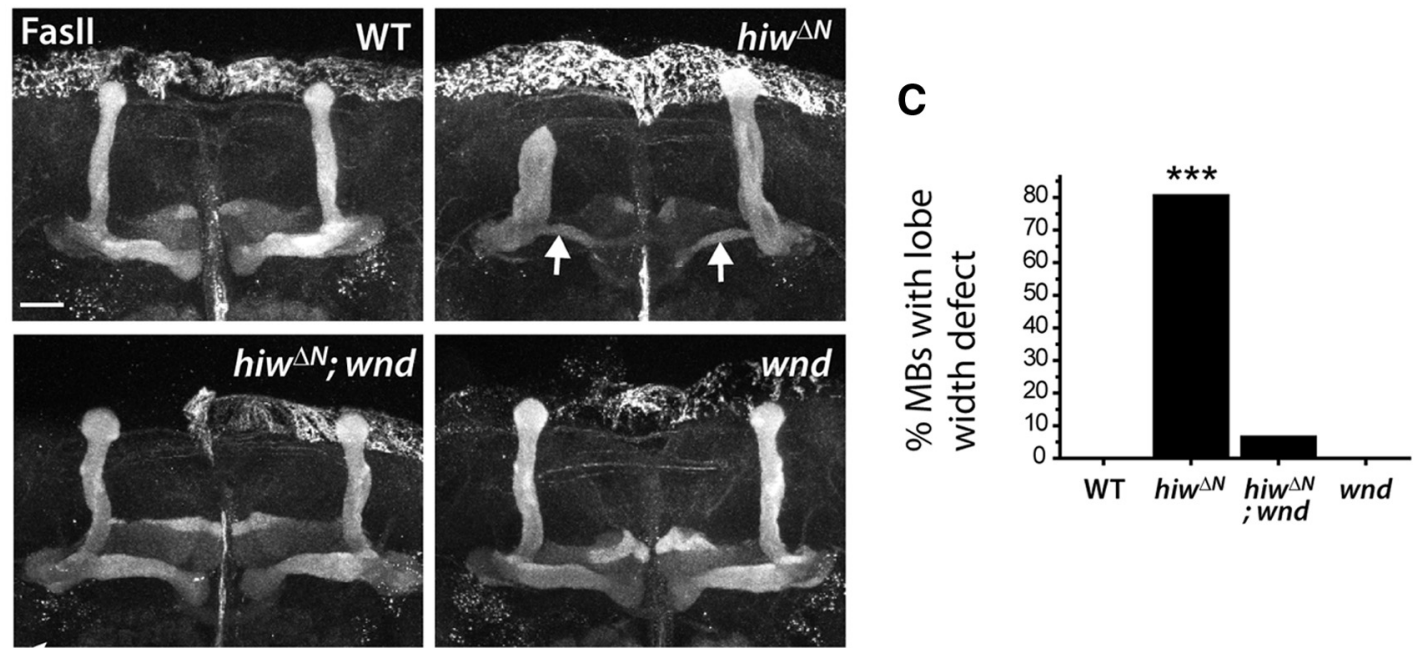

Figure 5. Failure to downregulate Wnd is responsible for the highwire MB phenotype. $A$, Immunostaining for Wnd in WT and hiw ${ }^{\Delta N} M B$ is shown in the confocal cross-sections of the third-instar larval brains. MB axons are visualized with mCD8 -GFP expression (green) driven by 0K107-Gal4. Wnd (red) is rarely detected in the MB lobes or the peduncles (P) from WT but is highly enriched in the highwire mutant MB. In hiw ${ }^{\Delta N}$, strong immunoreactivity against Wnd was observed in the core axons in the lobes (yellow arrow), lobe endings (white arrow), and the core of the peduncles (arrowhead), although whole lobes overall display a high level of Wnd (middle column in $h_{i} w^{\Delta N}$ images). B, C, Suppression of the highwire MB phenotype by mutations in wnd. B, MBs from late pupal WT, hiw ${ }^{\Delta N}$, hiw ${ }^{\Delta N}$; wnd $\left(h_{i w}{ }^{\Delta N}\right.$; wnd $\left.d^{1} / w n d^{3}\right)$, and wnd $\left(w n d^{1} / w n d^{3}\right)$ animals were immunostained for Fas II for the analysis of MB lobe morphologies. Lobe width defect (arrow) was quantified in C for the genotypes listed in $\boldsymbol{B} . n=10,10,16$, and 16 for WT, hiw ${ }^{\Delta N}$, hiw ${ }^{\Delta N}$; wnd, and wnd, respectively. ${ }^{* * *} p<0.001$ versus WT and $h i w^{\Delta N}$; wnd. Scale bars, $20 \mu \mathrm{m}$.

cell- autonomous effect from the $\alpha^{\prime} / \beta^{\prime}$ lobe. To restore Highwire function selectively in the $\alpha^{\prime} / \beta^{\prime}$ neurons of highwire mutants, we used NP2748-Gal4 (Fig. 3C) because this driver is developmentally restricted to $\alpha^{\prime} / \beta^{\prime}$ neurons; driving mCD8-GFP with NP2748-Gal4 reveals that expression is restricted to $\alpha^{\prime} / \beta^{\prime}$ neurons from the third-instar to adult stages. Remarkably, expression of the highwire transgene in the $\alpha^{\prime} / \beta^{\prime}$ neurons of highwire mutants primarily rescues the guidance defect in the $\alpha / \beta$ projections. $\alpha^{\prime} / \beta^{\prime}$ neuronal transgenic expression leads to a substantial increase in the percentage of MBs with a normal width of $\alpha / \beta$ lobes (Fig. $6 A, B$; $p \ll 0.001$ ). The phenotype of short $\alpha$ lobes is also rescued by $\alpha^{\prime} / \beta^{\prime}$ neuronal expression of highwire (hiw ${ }^{\Delta N}$, $24 \%, n=62 ; \alpha^{\prime} / \beta^{\prime}$ neuronal rescue, 9\%, $\left.n=65 ; p<0.05\right)$. Transgenic expression of highwire in the WT background does not alter the organization of $\alpha / \beta$ lobes. These findings demonstrate that Highwire activity is non-cell-autonomously required in the $\alpha^{\prime} / \beta^{\prime}$ lobes for the development of $\alpha / \beta$ axons.

We next asked whether Highwire expression in the $\alpha / \beta$ neurons is also important. To test for cell autonomy, we performed a mosaic analysis by generating single $\alpha / \beta$ cell highwire mutant clones in an otherwise heterozygous and phenotypically normal background. As a result, mutant axons navigate normally into separate $\alpha$ and $\beta$ lobes (94\%, $n=36 ; p=1$ vs WT clones; Fig. 6C), demonstrating that the guidance choice of highwire mutant axons is determined by non-cell-autonomous factors. Collectively, we conclude that Highwire is required in the $\alpha^{\prime} / \beta^{\prime}$ lobes not for the guidance of $\alpha^{\prime} / \beta^{\prime}$ axons but rather for the expression of a noncell-autonomous mechanism by which $\alpha^{\prime} / \beta^{\prime}$ neurons build the proper environment for $\alpha / \beta$ axon guidance.

\section{Reducing Plexin A signaling enhances the}

\section{highwire phenotype}

Guidance signals shaping MB development include ligands, receptors, their downstream signaling components, and the regulators of cytoskeleton (Billuart et al., 2001; Ng et al., 2002; Wang et al., 2004; Zhan et al., 2004; Nicolas and Preat, 2005; Boyle et al., 2006; Goossens et al., 2011; Shimizu et al., 2011). Because Highwire affects axon guidance in the $\mathrm{MB}$ via a non-cell-autonomous mechanism, we asked whether Highwire does so by affecting a known guidance signal. To identify the signaling pathways that potentially interact with Highwire, we screened through a small set of genes that are involved in axon guidance and tested for 
A
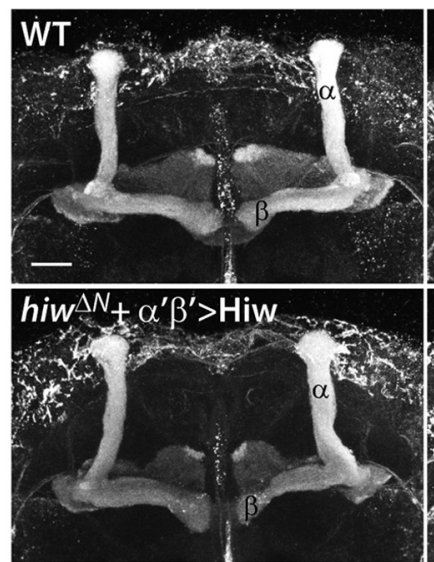

C

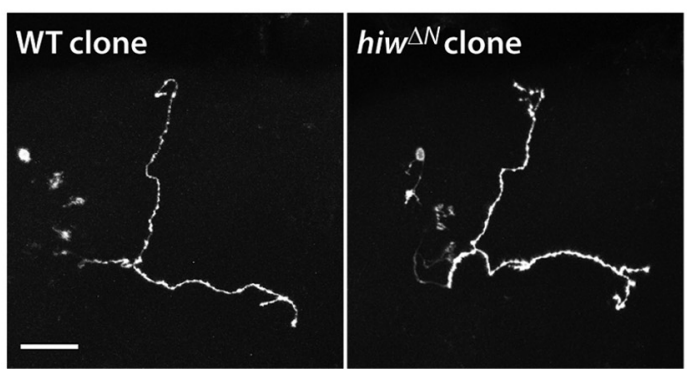

B
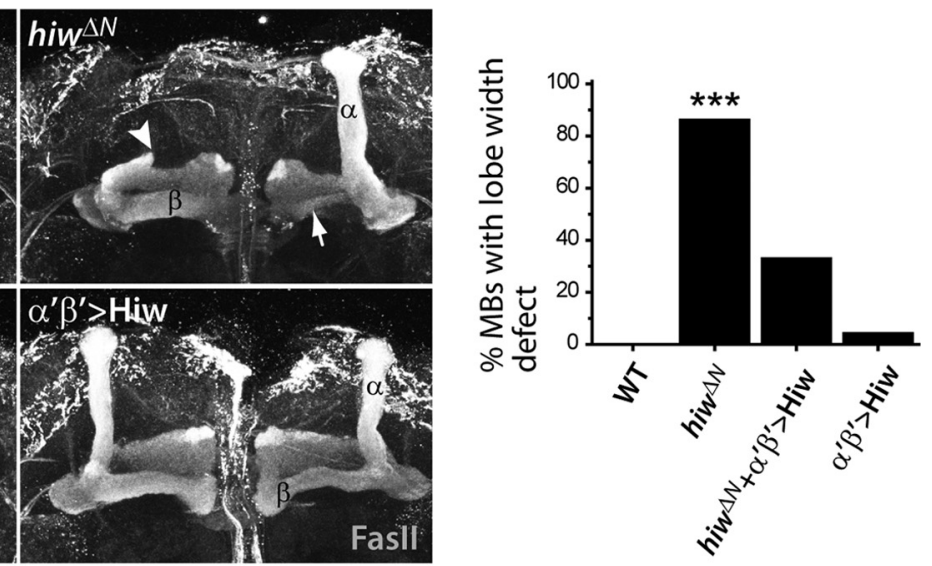

Figure 6. Non-cell-autonomous role of highwire in regulating the guidance of $\alpha / \beta$ lobe axons. $A$, The hiw ${ }^{\Delta N}$ phenotype in the $\alpha / \beta$ lobe structures (arrow and arrowhead) is largely rescued by expression of the highwire transgene with an $\alpha^{\prime} / \beta^{\prime}$ lobe driver, NP2748-Gal4. Anti-Fas II immunolabeling shows $\alpha / \beta$ lobes in WT, hiw ${ }^{\Delta N}$ (hiw ${ }^{\Delta N}{ }^{\prime N P 2748-G a l 4 / y), ~ h i w ~}{ }^{\Delta N}+\alpha^{\prime} \beta^{\prime}>$ Hiw (hiw ${ }^{\Delta N}, N P 2748-G a l 4 / y ;$ UAS-hiw/+), and $\alpha^{\prime} \beta^{\prime}>$ Hiw (NP2748-Gal4/y; UAS-hiw/+). $\boldsymbol{B}$, Histogram shows the results from $\boldsymbol{A}$ quantified for the percentages of MBs with lobe width defect in $\alpha / \beta$ lobes. WT, $n=42 ;$ hiw $^{\Delta N}, n=62 ; h_{i w^{\Delta N}}+\alpha^{\prime} \beta^{\prime}>$ Hiw,$n=65 ; \alpha^{\prime} \beta^{\prime}>$ Hiw, $n=28 ;{ }^{* *} p \ll 0.001$ versus WT and hiw ${ }^{\Delta N}+\alpha^{\prime} \beta^{\prime}>$ Hiw. C, Single $\alpha / \beta$ neuronal clones of WT or $h_{i w^{\Delta N}}$ were generated for mosaic analysis in phenotypically normal backgrounds. $\alpha / \beta$ axons of the hiw ${ }^{\Delta N}$ clones bifurcate and segregate normally. WT clones were induced in the MBs of hs-FLP,tub-Gal80,FRT19A/ FRT19A; UAS-mCD8-GFP/+; OK107-Gal4/+, whereas hiw ${ }^{\Delta N}$ clones were generated in hs-FLP,tub-Gal80,FRT19A/ hiw ${ }^{\Delta N}$,FRT19A; UAS-mCD8-GFP/+; OK107Gal4/+. The mosaic clones were labeled by GFP and immunostained with anti-GFP antibody. Scale bars, $20 \mu \mathrm{m}$.

mutations that enhance or suppress the MB defects in combination with a highwire mutant allele. Because guidance mutants are often recessive lethal, we examined animals heterozygous for these mutations in combination with a highwire allele. We used the hypomorphic hiw ${ }^{N D 8}$ allele because it has an intermediate phenotype and so has sufficient dynamic range to allow us to detect enhancement or suppression of the phenotype. Potential genetic interactions were determined by blinded scoring of MBs by categorizing the phenotypic severity of the Fas II staining pattern as normal, medium, or strong. The "strong" phenotypic category included MBs in which one lobe is nearly absent and the "medium" category included thinned lobes. The hiw ${ }^{N D 8}$ mutant has a phenotype consistent with the hypomorphic nature of this allele (Fig. $7 B$ ). We did not observe significant alteration of the hiw $^{N D 8}$ phenotype in combination with mutations in Dscam, slit, robo2, robo3, Ephrin, Pak, or dock. However, a mutation in plexA, a receptor for semaphorins, dominantly enhances the highwire phenotype (Fig. 7A). Removing one copy of plexin A has no MB defect on its own. However, when highwire is also mutated, loss of one copy of plexin A exacerbates the hiw ${ }^{N D 8}$ phenotype, approximately doubling the percentage of MBs with a strong phenotype (Fig. $7 B ; p<0.005$ ). This suggests that Plexin A signaling is impaired in the highwire mutant and that an additional modest disruption of the pathway by loss of one copy of plexin $A$ leads to significant loss of the guidance signal. In contrast, the highwire phenotype is not dominantly enhanced by loss of one copy of plexin $B$, a Plexin receptor for different semaphorin ligands, suggesting that Highwire modulates a specific Plexin A signal that promotes MB development. If this model were correct, then we would predict that a more severe disruption to Plexin A should also disrupt this pathway even in the presence of WT highwire. To test this hypothesis, we used transgenic RNAi to knockdown plexin $A$ in the MB. Consistent with the model, expression of plexin A-RNAi by OK107-Gal4 robustly disrupts MB lobe morphology, leading to abnormalities very similar to those seen in the highwire mutant, including thickening/thinning of $\alpha$ or $\beta$ lobes and the shortening of $\alpha$ lobes (Fig. 7C; $86 \%, n=22$ ). Hence, Plexin A signaling appears to be critical for $\mathrm{MB}$ axon development, and the Plexin A and Highwire pathways may interact to promote normal development of the MB.

We attempted to identify the relevant semaphorin in this system. We examined mutations or RNAi knockdown transgenes for Sema1a, a well-known ligand for Plexin A (Winberg et al., 1998), as well as Sema-5c, which has a short $\alpha$ lobe phenotype (Rollmann et al., 2007). We did not observe enhancement of the hiw ${ }^{N D 8}$ phenotype when inhibiting Sema-1a by one copy of Sema- $1 a^{k 13702}$ mutant allele or by Sema-1a-RNAi (VDRC104505) driven with OK107-Gal4. Likewise, a homozygous mutation in Sema-5c (Sema-5c ${ }^{B G 01245}$, viable) did not enhance hiw ${ }^{N D 8}$ phenotype. The tested semaphorin mutations (Sema-1a $a^{k 13702}$ heterozygotes and Sema-5c ${ }^{B G 01245}$ homozygotes) or the RNAi line display minimal or no MB defect on their own, in contrast to the severe phenotype caused by plexin $A-R$ $N A i$ (Fig. 7C). Hence, these semaphorins may not be involved in the Plexin A/Highwire signaling, their function may be redundant, or the tested reagents may not reduce semaphorin levels sufficiently. Despite the failure to implicate a specific semaphorin, our data do 
A

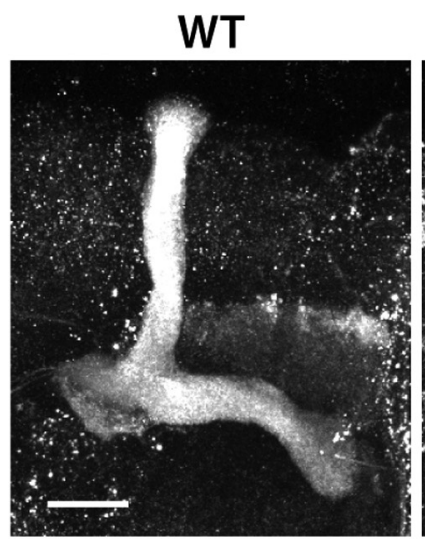

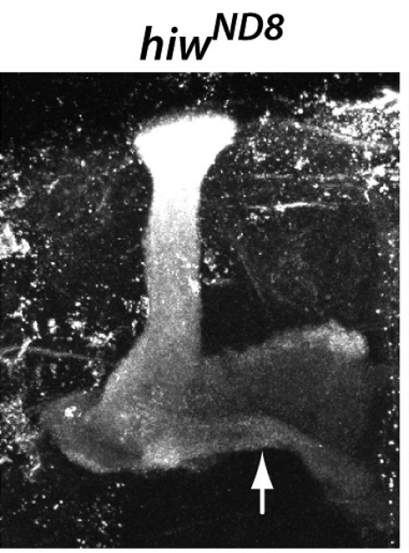

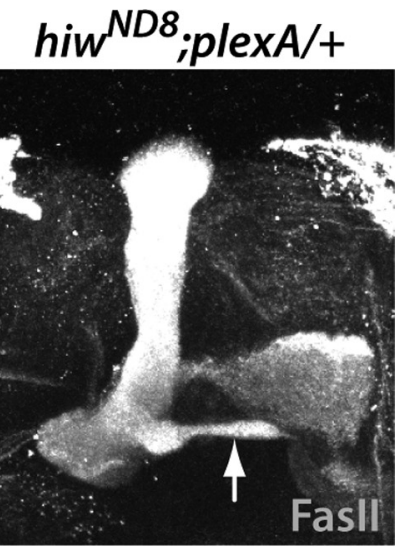

B
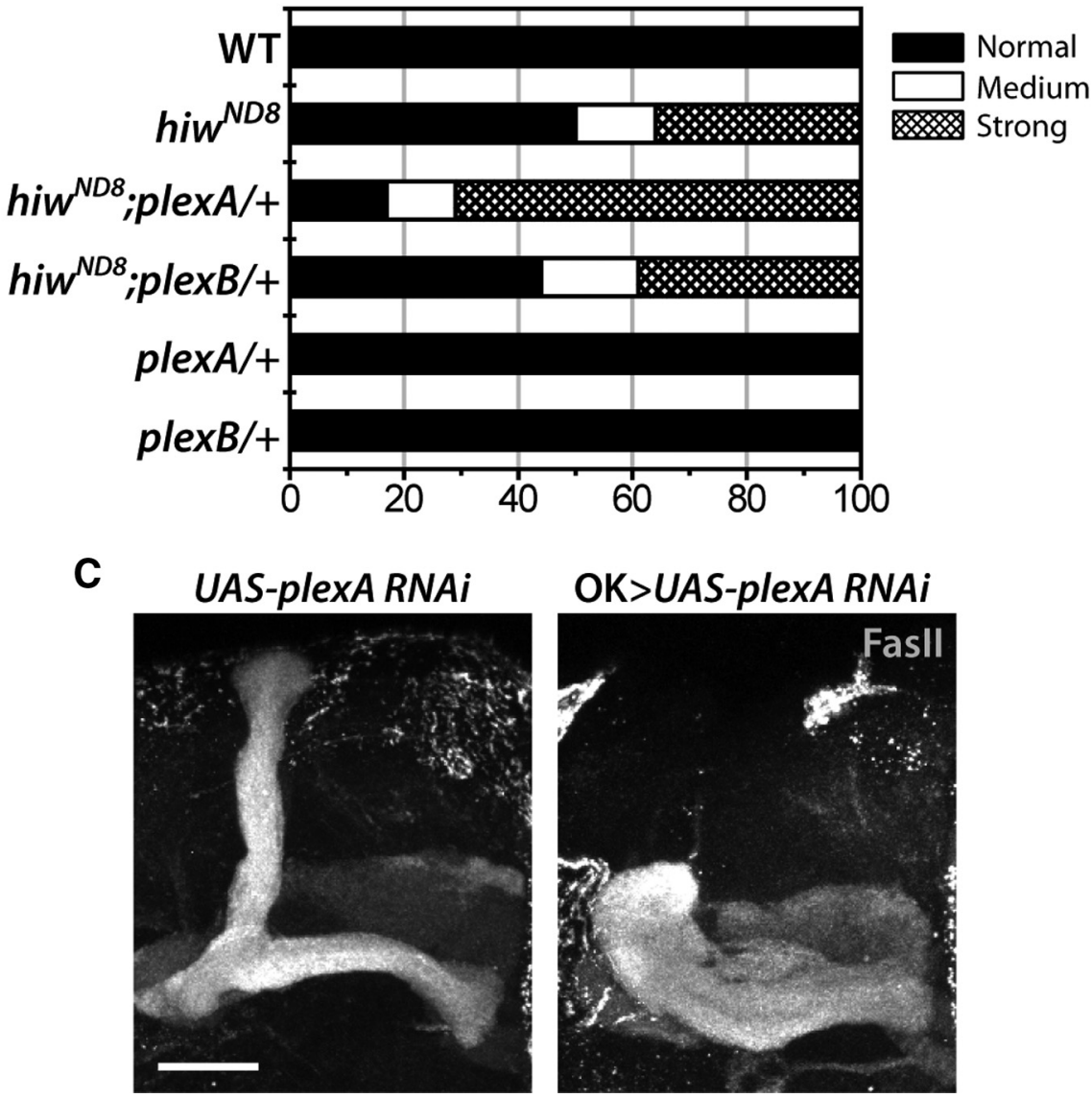

Figure 7. Genetic enhancement of the highwire $\alpha / \beta$ lobe phenotype by reducing Plexin A level. $A, B$, MBs from the genotypes listed in $\boldsymbol{B}$ were stained for Fas II for the analysis of $\alpha$ and $\beta$ lobe phenotype. Images taken from WT, hiw ${ }^{N 08}$, hiw ${ }^{N D 8}$; plexA/+ brains are shown in $A$. The lobe defects were categorized based on the severity of the phenotype. For example, each of left, middle, and right panels in $A$ shows an example of normal, medium, and strong phenotypes, respectively. The quantification is shown in $\boldsymbol{B}$ as the percentages of MBs in each category. The lobe defect is significantly enhanced in $h i w^{N D 8}$; plexA ++ compared with that in hiw $^{N D 8}(p<0.005) . n=8,36,42,18,10$, and 12 for WT, hiw ${ }^{N D 8}$, hiw ${ }^{N D 8}$; plexA/+, hiw ${ }^{N D 8}$; plexB/+, plexA/+, and plexB/+, respectively. C, Requirement of Plexin A signaling in the $\alpha / \beta$ lobe development. Expression of plexin A-RNAi by 0K107-Gal4 disrupts guidance of $\alpha / \beta$ axons as shown with anti-Fas II staining. Scale bars, $20 \mu \mathrm{m}$.

demonstrate a genetic interaction between Highwire and Plexin A in the developing MB. These findings support a model in which Highwire promotes Plexin A signaling, and this signaling mediates the proper axon guidance of $\alpha / \beta$ neurons via a non-cell-autonomous mechanism involving the pioneer axons of $\alpha^{\prime} / \beta^{\prime}$ neurons.

\section{Discussion}

Here we identify a requirement for the ubiquitin ligase highwire in $\mathrm{MB}$ development. In normal MB development, $\alpha / \beta$ neurons form axons that bifurcate and segregate into dorsal $\alpha$ and medial $\beta$ lobes of similar thickness. In the absence of highwire, the $\alpha / \beta$ axons bifurcate, but the sister branches fail to segregate into distinct lobes. Highwire regulates the guidance of these sister branches by limiting the levels of the MAPKKK Wnd/DLK. Here we demonstrate that highwire is required in the $\alpha^{\prime} / \beta^{\prime}$ neurons for the proper guidance of $\alpha / \beta$ axons, and genetic interactions suggest that this non-cell-autonomous guidance mechanism may involve Plexin A signaling.

Highwire is required for proper guidance of $\mathrm{MB}$ axons during development

Highwire is the Drosophila ortholog of an evolutionarily conserved family of ubiquitin ligases termed PHR proteins. Func- 
tional studies in worms, flies, fish, and mice have demonstrated roles for PHR proteins in synapse development and target selection as well as axon outgrowth, guidance, and regeneration (Schaefer et al., 2000; Wan et al., 2000; Zhen et al., 2000; Burgess et al., 2004; D'Souza et al., 2005; Bloom et al., 2007; Grill et al., 2007; Lewcock et al., 2007; Culican et al., 2009; Hammarlund et al., 2009; Hendricks and Jesuthasan, 2009; Yan et al., 2009; Xiong et al., 2010). In Drosophila, highwire is best studied for its role in restraining synaptic terminal growth at the NMJ. Studies in the fly neuromuscular system did not, however, find a role for highwire in motoneuron axon guidance (Wan et al., 2000). In the current study, we demonstrate that highwire does regulate axon guidance of MB neurons in Drosophila. The gross morphological defects, such as the short $\alpha$ lobe and thinning of either the $\alpha$ or $\beta$ lobe, present in the highwire MB lobes of the adult could be attributable to defects in either the development or maintenance of axons. However, we observe similar defects in both the developing and adult $\mathrm{MB}$, so the phenotype is not attributable to degeneration of previously formed axons. The defect is also inconsistent with a gross alteration in axon outgrowth or guidance. The $\alpha / \beta$ axons form, path-find appropriately through the peduncle, and branch at the appropriate location. Instead, the data suggest a selective deficit in responding to guidance cues at this choice point. After bifurcation of the axon, sister branches do not segregate into distinct lobes as in WT but rather travel together into the same lobe. This phenotype is consistent with loss of homotypic repulsion of sister branches and/or the inability to respond to selective guidance cues targeting the axons to particular lobes.

\section{Highwire promotes $\mathrm{MB}$ axon development by downregulating the Wnd/DLK MAPKKK}

In both fly and worm, PHR proteins sculpt synaptic terminals by restraining Wnd/DLK MAPKKK activity. In Drosophila, highwire acts as an ubiquitin ligase to limit the abundance of Wnd/DLK (Wu et al., 2005, 2007; Collins et al., 2006). Excess Wnd/DLK protein overactivates a MAP kinase signaling pathway that promotes synaptic terminal overgrowth. Here we demonstrate that highwire-dependent downregulation of Wnd/DLK is also required for segregation of sister branches of $\alpha / \beta$ axons and, hence, proper MB development. In the absence of highwire, levels of Wnd/DLK are elevated in the axons of the developing MB. Furthermore, genetic deletion of Wnd/DLK suppresses the highwiredependent phenotypes, demonstrating that Wnd/DLK is required for the aberrant behavior of $\alpha / \beta$ axons in the highwire mutant. We attempted to test whether overexpression of Wnd/DLK phenocopies highwire mutant MB by driving Wnd/DLK transgenic expression with OK107-Gal4 or MB subset Gal4 lines, including $\alpha^{\prime} / \beta^{\prime}$-specific NP2748-Gal4. However, the strong overexpression of Wnd/DLK resulted in either lethality or massive cell death in the MB, probably because of the excess activation of downstream JNK MAPK signaling (Whitfield et al., 2001; Besirli et al., 2005; Ghosh et al., 2011).

Although the relationship between the PHR ubiquitin ligase and DLK kinase is clear in flies and worms, studies in vertebrate systems paint a murkier picture. Analysis of PHR mutants in mice and zebrafish consistently demonstrate an important role in various aspects of axon development (Burgess et al., 2004; D'Souza et al., 2005; Bloom et al., 2007; Lewcock et al., 2007; Culican et al., 2009; Hendricks and Jesuthasan, 2009). However, the molecular mechanism of PHR action and the potential involvement of DLK in vertebrate axons is less clear. In cultured sensory axons from the Phr1 mutant magellan, axon morphology is disrupted and
DLK protein is mislocalized (Lewcock et al., 2007). In addition, pharmacological inhibition of p38, a MAP kinase that can be downstream of DLK, reduced the size of the abnormally large growth cones present in these mutant axons. These findings are consistent with a role for DLK activity in generating the Phr1dependent phenotypes (Lewcock et al., 2007). However, in an independently generated $\mathrm{Phrl}$ mutant, we observed no gross change in DLK levels and found that genetic deletion of DLK failed to suppress either corticothalamic axon guidance defects or motoneuron sprouting defects (Bloom et al., 2007). In zebrafish, mutations in the PHR ortholog esrom disrupt axon guidance and lead to an increase in JNK activation, consistent with a role for DLK, but inhibition of neither JNK nor p38 can suppress the esrom phenotypes, arguing against a functional role for DLK (Hendricks and Jesuthasan, 2009). Our finding that Wnd/DLK is essential for axonal phenotypes in Drosophila while it is dispensable for at least some axonal phenotypes in mice and fish suggests that there is no simple relationship between PHR targets and the cellular function of PHR proteins. PHR proteins do interact with a number of other proteins besides DLK, and so the mechanism of PHR-dependent axonal phenotypes is likely context dependent (Po et al., 2010).

Non-cell-autonomous function of Highwire in axon guidance During MB development, the $\alpha / \beta$ neurons are the last to be born and the last to extend their axons into the MB lobes (Lee et al., 1999). These $\alpha / \beta$ axons follow the path established by the earlierborn $\gamma$ and $\alpha^{\prime} / \beta^{\prime}$ axons. In the highwire mutant, the $\alpha / \beta$ axons form short, thin, or absent $\alpha / \beta$ lobes, whereas the $\gamma$ and $\alpha^{\prime} / \beta^{\prime}$ axons form morphologically normal lobes. Although such results would be consistent with a unique requirement for highwire in $\alpha / \beta$ neurons, a series of findings instead indicate that highwire is required in $\alpha^{\prime} / \beta^{\prime}$ neurons and indirectly affects the development of $\alpha / \beta$ axons via a non-cell-autonomous mechanism. First, in the highwire mutant, Wnd/DLK levels are elevated in $\gamma, \alpha^{\prime} / \beta^{\prime}$, and $\alpha / \beta$ axons, demonstrating that the Highwire ligase is likely targeting Wnd/DLK in all three cell types. Second, in the highwire mutant, the sister branches of the $\alpha / \beta$ axons fail to segregate but instead travel into the same lobe. However, within a brain hemisphere, most of the sister branches choose the same lobe, resulting in either a thickened $\alpha$ or $\beta$ lobe. Hence, the decision as to which lobe to enter is apparently not determined independently by each axon. Third, expression of highwire in the earlier-born $\alpha^{\prime} / \beta^{\prime}$ neurons is sufficient to rescue the defects in the $\alpha / \beta$ lobes. Fourth, in single-cell highwire $\alpha / \beta$ clones, sister axons segregate normally in an otherwise heterozygous background. Together, these data demonstrate a non-cell-autonomous requirement for highwire.

How might $\alpha^{\prime} / \beta^{\prime}$ axons affect the guidance decision of $\alpha / \beta$ axons? Misexpression of the cell adhesion molecule Fas II in the $\alpha^{\prime} / \beta^{\prime}$ neurons leads to the loss of either $\alpha$ or $\beta$ projections, demonstrating that inter-axonal interactions can affect $\alpha / \beta$ axon development and suggesting that $\alpha^{\prime} / \beta^{\prime}$ axons act as "pioneering axons" for the later-arriving $\alpha / \beta$ axons (Fushima and Tsujimura, 2007). Because the $\alpha^{\prime} / \beta^{\prime}$ axons form morphologically normal lobes in the highwire mutant, the defect is likely at the molecular level, potentially involving a change in either membraneassociated or secreted guidance cues. In the vertebrate CNS, the highwire ortholog Phrl is also required for a non-cellautonomous mechanism that guides cortical axons. In the absence of Phr1, cortical axons stall at the corticostriatal border and do not contribute to the internal capsule. In contrast, after conditional excision of Phrl exclusively in cortical neurons, these 
same cortical axons can now cross this choice point and path-find to the thalamus (Bloom et al., 2007). Hence, the requirement for PHR proteins for non-cell-autonomous axon guidance mechanisms is evolutionarily conserved, although we have no evidence that the molecular mechanism is conserved.

\section{Highwire promotes Plexin A signaling in MB development}

To investigate the molecular mechanism of the non-cellautonomous requirement for highwire, we tested for genetic interactions between highwire and candidate guidance molecules. Our data suggest that Highwire promotes a Plexin A signaling mechanism that is required for proper $\alpha / \beta$ lobe development. Loss of a single copy of the plexin $A$ gene has no effect on $\mathrm{MB}$ development in an otherwise WT background but enhances the phenotype of a weak highwire allele. Furthermore, RNAimediated knockdown of plexin $A$ in the $\mathrm{MB}$ has a very similar phenotype to loss of highwire, with abnormal thickness of $\alpha / \beta$ lobes and shortened $\alpha$ lobes. Hence, Plexin A is required for normal MB development. Plexins are receptors for semaphorins (Winberg et al., 1998; Zhou et al., 2008), and both Sema-1a and Sema- $5 c$ are required for normal MB development (Komiyama et al., 2007; Rollmann et al., 2007). Our genetic studies did not uncover a genetic interaction between either of these semaphorins and highwire, but the absence of such an interaction does not rule out the involvement of these or other semaphorins. Two potential models are consistent with these genetic interaction studies. First, Plexin A may function to downregulate Wnd/DLK, potentially via inhibition of Rac GTPase signaling (Liu and Strittmatter, 2001; Xu et al., 2001). In the absence of either plexin $A$ or highwire, Wnd/DLK activity would be upregulated, disrupting axonal interactions between $\alpha^{\prime} / \beta^{\prime}$ axons and $\alpha / \beta$ axons via unknown mechanisms. Alternatively, excess Wnd/DLK activity in the highwire mutant could disrupt the Plexin A signaling pathway that is necessary for $\alpha / \beta$ lobe development. The mechanisms by which Highwire and Plexin A signaling converge will be the subject of future studies.

\section{References}

Araújo SJ, Tear G (2003) Axon guidance mechanisms and molecules: lessons from invertebrates. Nat Rev Neurosci 4:910-922.

Besirli CG, Wagner EF, Johnson EM Jr (2005) The limited role of NH2terminal c-Jun phosphorylation in neuronal apoptosis: identification of the nuclear pore complex as a potential target of the JNK pathway. J Cell Biol 170:401-411.

Billuart P, Winter CG, Maresh A, Zhao X, Luo L (2001) Regulating axon branch stability: the role of p190 RhoGAP in repressing a retraction signaling pathway. Cell 107:195-207.

Bloom AJ, Miller BR, Sanes JR, DiAntonio A (2007) The requirement for Phr1 in CNS axon tract formation reveals the corticostriatal boundary as a choice point for cortical axons. Genes Dev 21:2593-2606.

Boyle M, Nighorn A, Thomas JB (2006) Drosophila Eph receptor guides specific axon branches of mushroom body neurons. Development 133:1845-1854.

Burgess RW, Peterson KA, Johnson MJ, Roix JJ, Welsh IC, O’Brien TP (2004) Evidence for a conserved function in synapse formation reveals $\mathrm{Phrl}$ as a candidate gene for respiratory failure in newborn mice. Mol Cell Biol 24:1096-1105.

Collins CA, Wairkar YP, Johnson SL, DiAntonio A (2006) Highwire restrains synaptic growth by attenuating a MAP kinase signal. Neuron 51:57-69.

Culican SM, Bloom AJ, Weiner JA, DiAntonio A (2009) Phrl regulates retinogeniculate targeting independent of activity and ephrin-A signalling. Mol Cell Neurosci 41:304-312.

Davis RL (2005) Olfactory memory formation in Drosophila: from molecular to systems neuroscience. Annu Rev Neurosci 28:275-302.

D'Souza J, Hendricks M, Le Guyader S, Subburaju S, Grunewald B, Scholich K, Jesuthasan S (2005) Formation of the retinotectal projection requires
Esrom, an ortholog of PAM (protein associated with Myc). Development 132:247-256.

Fahrbach SE (2006) Structure of the mushroom bodies of the insect brain. Annu Rev Entomol 51:209-232.

Fushima K, Tsujimura H (2007) Precise control of fasciclin II expression is required for adult mushroom body development in Drosophila. Dev Growth Differ 49:215-227.

Ghosh AS, Wang B, Pozniak CD, Chen M, Watts RJ, Lewcock JW (2011) DLK induces developmental neuronal degeneration via selective regulation of proapoptotic JNK activity. J Cell Biol 194:751-764.

Goossens T, Kang YY, Wuytens G, Zimmermann P, Callaerts-Végh Z, Pollarolo G, Islam R, Hortsch M, Callaerts P (2011) The Drosophila L1CAM homolog Neuroglian signals through distinct pathways to control different aspects of mushroom body axon development. Development 138:1595-1605.

Grill B, Bienvenut WV, Brown HM, Ackley BD, Quadroni M, Jin Y (2007) C. elegans RPM-1 regulates axon termination and synaptogenesis through the Rab GEF GLO-4 and the Rab GTPase GLO-1. Neuron 55:587-601.

Hammarlund M, Nix P, Hauth L, Jorgensen EM, Bastiani M (2009) Axon regeneration requires a conserved MAP kinase pathway. Science 323:802-806

Heisenberg M (1998) What do the mushroom bodies do for the insect brain? An introduction. Learn Mem 5:1-10.

Hendricks M, Jesuthasan S (2009) PHR regulates growth cone pausing at intermediate targets through microtubule disassembly. J Neurosci 29:6593-6598.

Komiyama T, Sweeney LB, Schuldiner O, Garcia KC, Luo L (2007) Graded expression of semaphorin-1a cell-autonomously directs dendritic targeting of olfactory projection neurons. Cell 128:399-410.

Lee T, Luo L (1999) Mosaic analysis with a repressible cell marker for studies of gene function in neuronal morphogenesis. Neuron 22:451-461.

Lee T, Lee A, Luo L (1999) Development of the Drosophila mushroom bodies: sequential generation of three distinct types of neurons from a neuroblast. Development 126:4065-4076.

Lewcock JW, Genoud N, Lettieri K, Pfaff SL (2007) The ubiquitin ligase Phrl regulates axon outgrowth through modulation of microtubule dynamics. Neuron 56:604-620

Liu BP, Strittmatter SM (2001) Semaphorin-mediated axonal guidance via Rho-related G proteins. Curr Opin Cell Biol 13:619-626.

Nakata K, Abrams B, Grill B, Goncharov A, Huang X, Chisholm AD, Jin Y (2005) Regulation of a DLK-1 and p38 MAP kinase pathway by the ubiquitin ligase RPM-1 is required for presynaptic development. Cell 120:407-420.

Ng J, Nardine T, Harms M, Tzu J, Goldstein A, Sun Y, Dietzl G, Dickson BJ, Luo L (2002) Rac GTPases control axon growth, guidance and branching. Nature 416:442-447.

Nicolas E, Preat T (2005) Drosophila central brain formation requires Robo proteins. Dev Genes Evol 215:530-536.

Po MD, Hwang C, Zhen M (2010) PHRs: bridging axon guidance, outgrowth and synapse development. Curr Opin Neurobiol 20:100-107.

Rallis A, Moore C, Ng J (2010) Signal strength and signal duration define two distinct aspects of JNK-regulated axon stability. Dev Biol 339:65-77.

Rollmann SM, Yamamoto A, Goossens T, Zwarts L, Callaerts-Végh Z, Callaerts P, Norga K, Mackay TF, Anholt RR (2007) The early developmental gene Semaphorin $5 \mathrm{c}$ contributes to olfactory behavior in adult Drosophila. Genetics 176:947-956.

Schaefer AM, Hadwiger GD, Nonet ML (2000) rpm-1, a conserved neuronal gene that regulates targeting and synaptogenesis in C. elegans. Neuron 26:345-356.

Shimizu K, Sato M, Tabata T (2011) The wnt5/planar cell polarity pathway regulates axonal development of the Drosophila mushroom body neuron. J Neurosci 31:4944-4954.

Tanaka NK, Tanimoto H, Ito K (2008) Neuronal assemblies of the Drosophila mushroom body. J Comp Neurol 508:711-755.

Wan HI, DiAntonio A, Fetter RD, Bergstrom K, Strauss R, Goodman CS (2000) Highwire regulates synaptic growth in Drosophila. Neuron 26:313-329.

Wang J, Zugates CT, Liang IH, Lee CH, Lee T (2002) Drosophila Dscam is required for divergent segregation of sister branches and suppresses ectopic bifurcation of axons. Neuron 33:559-571.

Wang J, Ma X, Yang JS, Zheng X, Zugates CT, Lee CH, Lee T (2004) Transmembrane/juxtamembrane domain-dependent Dscam distribution and 
function during mushroom body neuronal morphogenesis. Neuron 43:663-672.

Whitfield J, Neame SJ, Paquet L, Bernard O, Ham J (2001) Dominantnegative c-Jun promotes neuronal survival by reducing BIM expression and inhibiting mitochondrial cytochrome c release. Neuron 29:629-643.

Winberg ML, Noordermeer JN, Tamagnone L, Comoglio PM, Spriggs MK, Tessier-Lavigne M, Goodman CS (1998) Plexin A is a neuronal semaphorin receptor that controls axon guidance. Cell 95:903-916.

Wu C, Wairkar YP, Collins CA, DiAntonio A (2005) Highwire function at the Drosophila neuromuscular junction: spatial, structural, and temporal requirements. J Neurosci 25:9557-9566.

Wu C, Daniels RW, DiAntonio A (2007) DFsn collaborates with Highwire to down-regulate the Wallenda/DLK kinase and restrain synaptic terminal growth. Neural Dev 2:16.

Wu JS, Luo L (2006) A protocol for mosaic analysis with a repressible cell marker (MARCM) in Drosophila. Nat Protoc 1:2583-2589.

Xiong X, Wang X, Ewanek R, Bhat P, Diantonio A, Collins CA (2010) Pro- tein turnover of the Wallenda/DLK kinase regulates a retrograde response to axonal injury. J Cell Biol 191:211-223.

Xu Z, Maroney AC, Dobrzanski P, Kukekov NV, Greene LA (2001) The MLK family mediates c-Jun N-terminal kinase activation in neuronal apoptosis. Mol Cell Biol 21:4713-4724.

Yan D, Wu Z, Chisholm AD, Jin Y (2009) The DLK-1 kinase promotes mRNA stability and local translation in C. elegans synapses and axon regeneration. Cell 138:1005-1018.

Zhan XL, Clemens JC, Neves G, Hattori D, Flanagan JJ, Hummel T, Vasconcelos ML, Chess A, Zipursky SL (2004) Analysis of Dscam diversity in regulating axon guidance in Drosophila mushroom bodies. Neuron 43:673-686.

Zhen M, Huang X, Bamber B, Jin Y (2000) Regulation of presynaptic terminal organization by C. elegans RPM-1, a putative guanine nucleotide exchanger with a RING-H2 finger domain. Neuron 26:331-343.

Zhou Y, Gunput RA, Pasterkamp RJ (2008) Semaphorin signaling: progress made and promises ahead. Trends Biochem Sci 33:161-170. 